\title{
Opalimosina (Diptera: Sphaeroceridae) from China with descriptions of two new species
}

\author{
Li-Xin Su, Guang-Chun Liu* \& Jie Xu
}

\begin{abstract}
Su, L.-X., Liu, G.-C. \& Xu, J. 2013: Opalimosina (Diptera: Sphaeroceridae) from China with descriptions of two new species. - Entomol. Fennica. 24: 94 99.

Two new species of the genus Opalimosina Roháček, namely Opalimosina (O.) differentialis $\mathrm{Su}, \mathbf{s p}$. n. and Opalimosina (O.) prominentia $\mathrm{Su}, \mathbf{s p}$. n., are described from China. A key to the world species of the subgenus Opalimosina is given.

L.-X. Su \& G.-C. Liu (*corresponding author), Liaoning Key Laboratory of Urban Integrated Pest Management and Ecological Security, Shenyang University, Shenyang, Liaoning 110044, China; Corresponding author's e-mail:liugc@yahoo.cn

J. Xu, Shenyang Normal University, Shenyang, Liaoning 110034, China
\end{abstract}

Received 2 September 2012, accepted 21 December 2012

\section{Introduction}

Opalimosina Roháček (1983) is reliably identified by the following features: postvertical setae are always present, hind tibia are with a distinct ventral spine in many species (the subgenus Opalimosina), and the basiphallus is with a large epiphallus.

Species of the genus occur in all zoogeographic regions of the world except in the Afrotropical and Antarctic Regions. Presently, it includes thirteen species, ten Palaearctic, six Oriental, one Nearctic, one Neotropical and one Australasian/Oceanian (Roháček et al. 2001, Hayashi 2009, Hayashi 2010, Marshall et al. 2011). Except for Su's (2011) report of Opalimosina (O.) mirabilis (Collin) and Opalimosina (O.) pseudomirabilis Hayashi from China, little is known of the Chinese species of this genus. Four species are reported from China in this study, of which Opalimosina (O.) differentialis and Opalimosina (O.) prominentia are new to science. A key to the world species of the subgenus Opalimosina is given.

\section{Material and methods}

The specimens were collected with sweep nets and Malaise traps in coniferous and broad-leaved mixed forests from mountains of Jiangxi Province, Shanxi Province, Sichuan Province and Yunnan Province, China, and preserved in $80 \%$ ethyl alcohol in vials. The whole abdomen was detached and left in 10\% KOH until semi-transparent, rinsed in water and then the whole abdomen placed into a cavity slide with glycerol for further dissection and examination, finally preserved in capsule with glycerin. The illustrations of genitalia and legs were made using a Leica DM 2000 compound microscope fitted with a drawing tube.

Voucher specimens including types are deposited in the collection of Liaoning Key Laboratory of Urban Integrated Pest Management and 
Ecological Security, Shenyang University, Shenyang, Liaoning Province, China. Morphological terminology follows Smith \& Marshall (2004).

\section{Taxonomy}

\subsection{Opalimosina (O.) mirabilis (Collin, 1902).}

Limosina mirabilis Collin, 1902: 59.

Opalimosina (Opalimosina) mirabilis: Su, 2011: 80-82, 188.

Material examined. 2 ๙ิे, China: Sichuan, Gonggashan, $101^{\circ} 52$ 35'"E, $29^{\circ} 3541^{\prime \prime} \mathrm{N}, 1,500$ m, 2.VIII.2004, sweep nets, G.-C. Liu leg.

Distribution. Europe, Australia, New Zealand, Hawaii (USA), Canada, USA, Argentina, Brazil, Colombia, Costa Rica, Mexico, China (Sichuan), Cyprus, Iran, Japan, Nepal (?), North Korea, Mongolia, Pakistan, Tunisia, Turkey.

\subsection{Opalimosina (O.) pseudomirabilis Hayashi, 1989.}

Opalimosina (Opalimosina) pseudomirabilis Hayashi, 1989: 63.

Opalimosina (Opalimosina) pseudomirabilis: Su, 2011: 82-83, 189.

Material examined. 1 ふै, China: Jiangxi, Jian, Shuangjiang, $114^{\circ} 59$ 34"E, 276 46”N, 174 m, 1.VI.2009, Malaise traps, L.-L. Yi and Y. Li leg. 2 ภेग, 1 ㅇ, China: Yunnan, Tengchong, Guyong, $98^{\circ} 16$ 39'E, $25^{\circ} 19$ 13'N, 1,750-1,920 m, 8.VIII.2009, sweep nets, L.-X. Su and J.-F. Wang leg. 1 ๙ , China: Jiangxi, Jian, Shuangjiang, $114^{\circ} 59$ 34'”, $27^{\circ} 6$ 46”N, 174 m, 10.V.2009, Malaise traps, L.-L. Yi and Y. Li leg.

Distribution. China (Jiangxi, Yunnan), Nepal, Pakistan.

\subsection{Opalimosina (O.) differentialis $\mathrm{Su}$, sp. n.} (Fig. 1)

Type material. Holotype $\sigma^{\top}$ : China: Shanxi, Qinshui, Zhongcun, Xiachuan, $112^{\circ} 00$ 55.8'E, $35^{\circ} 2631.3$ 'N, 1,686 m, 19.7.2012, sweep nets, L.-X. Su leg. Paratype ỡ: China: Sichuan,
Gonggashan, 10152 35’'E, $29^{\circ} 35$ 41'N, 1,500 m, 2.VIII.2004, sweep nets, G.-C. Liu leg.

Description. Male. Body length approx. 1.5 $\mathrm{mm}$, wing length approx. $1.1 \mathrm{~mm}$. General color brownish black, including head, thorax, abdomen and legs; antennae also brownish black; halter with stem pale brown, knob brown.

Head. 2 orbital setae, with additional setulae below and inside orbitals. Postvertical setae small and cruciate. Interfrontalia with 3 subequal setae. Eye height 2.6 times as long as genal height. Genal seta small. Aristal hairs twice as long as basal width of arista.

Thorax. Thoracic chaetotaxy: 1 long postpronotal seta; 2 dorsocentral setae, the anterior indistinct; 8 rows of acrostichal setulae in front of suture. Katepisternum with small anterodorsal hair and large posterodorsal seta. Scutellum with 2 pairs of marginal setae. Mid tibia (Fig. 1a) proximally with a short anterodorsal seta, distally with a short anterodorsal and a long dorsal and a short posterodorsal seta; posterodorsally with a row of small setulae; ventroapical seta long; with short anteroventral seta below middle. Hind tibia (Fig. 1b) with a curved preapical ventral spine, 0.25 times as long as hind tibia.

Wing pale brown, veins brown. $\mathrm{C}$ extending to $\mathrm{R}_{4+5}$, first costal sector basally with the usual 2 setae. Second costal sector 1.2 times as long as the third, crossveins $\mathrm{r}-\mathrm{m}$ and $\mathrm{dm}-\mathrm{cu}$ separated by twice the length of dm-cu. $\mathrm{R}_{2+3}$ distinctly sinuate, apically very slightly bent up to $C$. $R_{4+5}$ distinctly bent up to $\mathrm{C}$. Discal cell with stump veins of $\mathrm{M}$ and $\mathrm{CuA}_{1}$. Anal vein almost straight and reduced. Alula small and narrow, apically slightly blunt.

Male abdomen. Syntergite 1+2 large, about twice as long as tergite 3 at middle, subequal in width to the latter. Synsternite $1+2$ pale, sternites 3-4 uniformly dark. Sternite 5 (Fig. 1g) with a pale and narrow area in the middle, a row of 6 short spine-like setae posterior to the pale area; posteromedially with a deep and narrow incision, each side composed of a row of comb-like spines and a setulose projection, inner row of spines situated above outer setulose projection. Sternite 6 simple. Epandrium (Fig. 1e, d) with short and sparse setulae. Cercus (Fig. 1e) simple. Surstylus (Fig. 1f) with long ventral setae, 2 thick and long posteroventral setae, and small dorsal or lateral setulae; anterior part slightly narrow, with a 

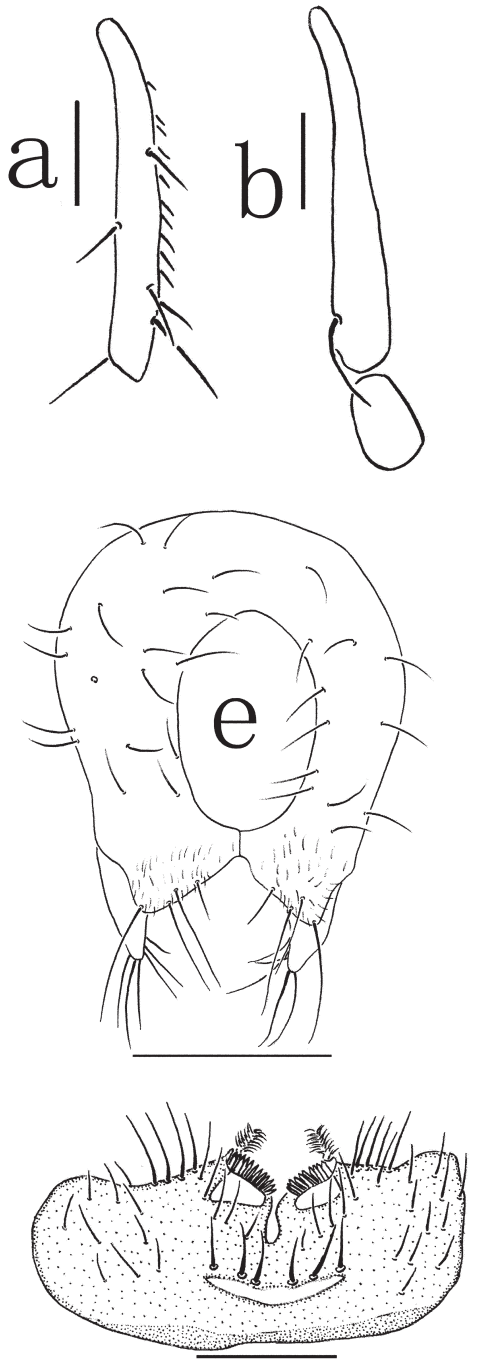

g
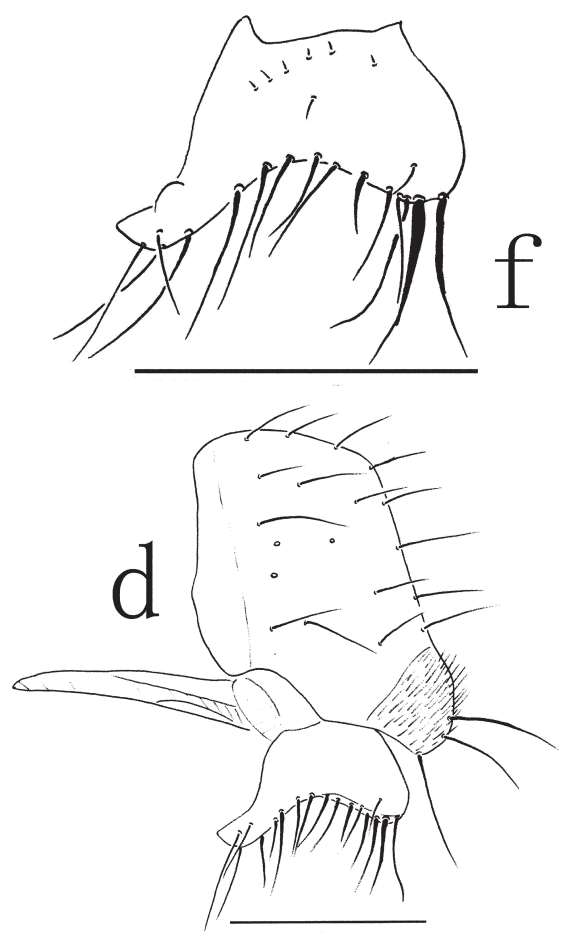

Fig. 1. Opalimosina differentialis Su, sp. $\mathbf{n}$, holotype male, from Shanxi, China. - a. Mid tibia, anteriorly. $-b$. Hind tibia, anteriorly. - c. Aedeagus and associated parts, laterally. -d. Genitalia laterally. - e. Genitalia caudally. $-\mathrm{f}$. Surstylus laterally. - g. Sternite 5 ventrally. Scale bars $0.1 \mathrm{~mm}$. small, blunt, preapical dorsal convex ridge. Epiphallus (Fig. 1c) straight, short and thick. Postgonite (Fig. 1c) slender, distinctly sinuate, with a slightly long, preapical posterior setula, and a short, anterior setula on apical third. Distiphallus (Fig. 1c) with a pair of dorsal projections.

Female. Unknown.

Distribution. China (Shanxi, Sichuan).

Etymology. The specific name is derived from the characteristic male sternite 5 with 2 paired, differentiated, posteromedial lobes.

Remarks. In Roháček's (1983) key to European species and Hayashi's (2010) key to Japanese species, this new species runs to Opali- mosina (O.) monticola Hayashi. However, male sternite 5 and postgonite are very different. The new species can be easily distinguished from the other members of the genus by the characteristics of sternite 5 described above.

\subsection{Opalimosina (O.) prominentia $\mathrm{Su}$, sp. n.}

(Fig. 2)

Type material. Holotype $\widehat{\jmath}$ : China: Shanxi, Qinshui, Zhongcun, Xiachuan, $112^{\circ} 00$ 55.8”E, $35^{\circ} 2631.3$ ”N, 1,686 m, sweep nets, 19.VII.2012, L.-X. Su leg.

Description. Male. Body length approx. 1.3 
Fig. 2. Opalimosina prominentia Su, sp. n., holotype male, from Shanxi, China. - a. Mid tibia, anteriorly. $-b$. Hind tibia, anteriorly. - c. Aedeagus and associated parts, laterally. - d. Genitalia laterally. - e. Genitalia caudally. $-\mathrm{f}$. Sternites 4 and 5 ventrally. Scale bars 0.1 $\mathrm{mm}$.
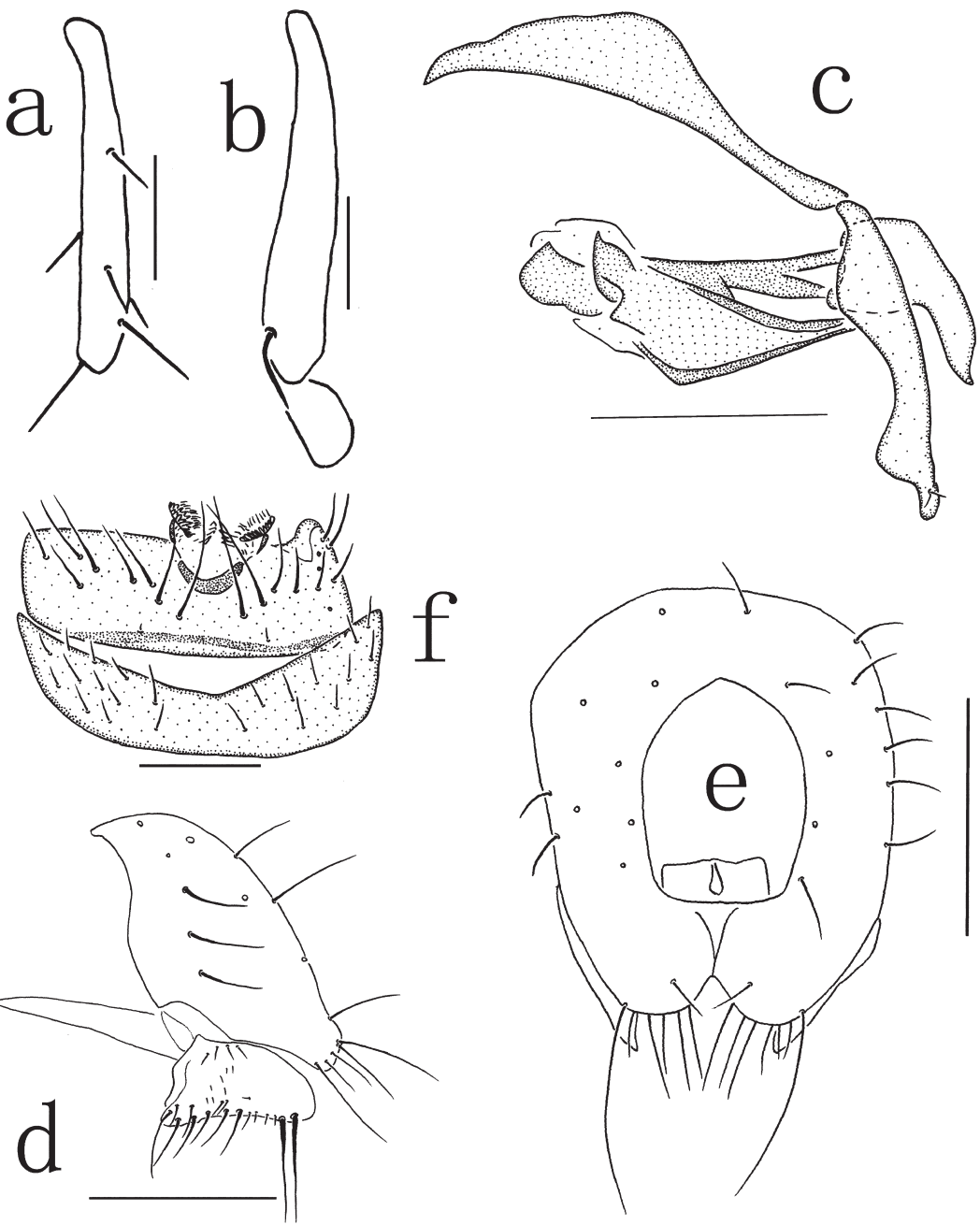

mm, wing length approx. $1.0 \mathrm{~mm}$. General color brownish black, including head, thorax, abdomen, legs and antennae; halter with stem and knob brown.

Head. 2 orbital setae, with additional setulae below and inside orbitals. Postvertical setae small and cruciate. Interfrontalia with 3 subequal setae. Eye height twice as long as genal height. Genal seta small. Aristal hairs 1.5 times as long as basal width of arista.

Thorax. Thoracic chaetotaxy: 1 long postpronotal seta; 2 dorsocentral setae, anterior one indistinct; 6 rows of acrostichal setulae in front of suture. Katepisternum with anterodorsal small setula and posterodorsal large seta. Scutellum with 2 pairs of marginal setae. Mid tibia (Fig. 2a) proximally with a short anterodorsal seta (slightly longer than distal posterodorsal seta, subequal to distal anterodorsal seta), distally with a short anterodorsal and a long dorsal and a short posterodorsal seta; ventroapical seta long; with short anteroventral seta below middle. Hind tibia (Fig. 2b) with a curved, preapical ventral spine, 0.2 times as long as hind tibia.

Wing pale brown, veins brown. $\mathrm{C}$ extending to $\mathrm{R}_{4+5}$, first costal sector basally with the usual 2 setae. Second costal sector 1.2 times as long as the third, crossveins $r-m$ and dm-cu separated by 2.8 times the length of dm-cu. $R_{2+3}$ distinctly sinuate, apically very slightly bent up to $\mathrm{C}$. $\mathrm{R}_{4+5}$ slightly bent up to $C$. Discal cell with stump veins of $M$ and $\mathrm{CuA}_{1}$. Anal vein almost straight and reduced. Alula small and narrow, apically slightly blunt.

Male abdomen. Syntergite 1+2 large, about 
twice as long as tergite 3 at middle, subequal in width to the latter. Synsternite $1+2$ anteromedially pale, sternites 3 and 4 uniformly pigmented. Sternite 4 (Fig. 2f) with posterior margin largely wedge-shaped. Sternite 5 (Fig. 2f) with sparse and short setae on posterolateral area and 4 long setae in the middle; a dark, moon-like, posteromedial area, each side on posteromedial margin with a dark, small process connecting to a row of comb-like spines. Sternite 6 simple. Epandrium (Fig. 2e, d) with sparse and short setulae. Cercus (Fig. 2e) simple, not protruded ventrally. Surstylus (Fig. 2d) triangular, with short setulae, 2 long and thick posteroventral setae, 4 short dorsal setulae. Epiphallus (Fig. 2c) short, thick and straight. Postgonite (Fig. 2c) with 2 large anterior broad lobes, a short posteropreapical setula. Distiphallus (Fig. 2c) with a pair of dorsal projections and a pair of apical sclerites.

Female. Unknown.

Distribution. China (Shanxi).

Etymology. The specific epithet refers to the postgonite, which has a sinuate anterior margin including two broad lobes.

Remarks. This new species is most similar with Opalimosina (O.) calcarifera (Roháček) and Opalimosina (O.) spathulata Hayashi in the preapical ventral spine of hind tibia, wing vein $\mathrm{C}$ not extending beyond $\mathrm{R}_{4+5}$, the shape and ventral setae of surstylus. It differs from the latter two by the characteristic male sternite 5 , the shape of cercus and anteriorly sinuate postgonite. The male sternite 5 and postgonite are particularly distinctive.

\subsection{Key to the world species of the subgenus Opalimosina}

1. Hind tibia with a small preapical ventral spine or without any long preapical ventral spine 2

- Hind tibia with a large preapical ventral spine, at least $1 / 5$ as long as hind tibia

2. Male sternite 5 (Papp 1991: Fig. 34) without any distinct pale area on disc, with long posterolateral setae and 2 groups of posteromedial setae, posteromedial margin with a process bearing 4 pairs of short setae

stepheni Papp

- Male sternite 5 with a pale area on disc, with short lateral and posteromedial setae, posteromedial margin with one or two rows of comblike short spines and one or two projections on each side

3. Male sternite 5 (Roháček 1983: Fig. 1069) with a pale arc-shaped stripe and a wide and deep posteromedial incision; each side of posteromedial margin composed of a row of comb-like short spines and a thin internal projection collini (Richards)

- Male sternite 5 (Roháček 1983: Fig. 1055) with a pale rhomb-like area in the middle, without posteromedial incision, each side of posteromedial margin composed of two rows of comb-like short spines and two thin external projections simplex (Richards)

4. Hind tibia with preapical ventral spine at least $1 / 3$ as long as hind tibia 5

- Hind tibia with preapical ventral spine less than $1 / 3$ as long as hind tibia

6

5. Wing vein $\mathrm{C}$ extending to $\mathrm{R}_{4+5}$; male sternite 5 (Hayashi 1989: Fig. 4) with a pale, narrow, rather deep medial area, with sparse and short setulae on anterior and lateral margins; surstylus (Hayashi 1989: Fig. 5) arc-shaped, posteroventral margin with 2 short and thick setae pseudomirabilis (Hayashi)

- Wing vein $\mathrm{C}$ extending beyond $\mathrm{R}_{4+5}$; male sternite 5 (Roháček 1983: Fig. 1034) with 7 posterior wide and 1 anteromedial very narrow pale areas, lateral area with short setulae and 2 groups of long setulae; surstylus (Roháček 1983: Fig. 1031) triangular, posteroventral margin with 3 long and thick setae mirabilis (Collin)

6. Male sternite 5 without a posteromedial incision

- Male sternite 5 with a deep posteromedial incision

8

7. Male sternite 5 (Fig. 2f) with a dark, moonlike posteromedial part and a dark, narrow anterior stripe, 4 long setae in front of dark moon-like part, posterolateral part with sparse and short setae; postgonite (Fig. 2c) with 2 anterior broad lobes prominentia $\mathrm{Su}, \mathbf{s p . n}$.

- Male sternite 5 (Hayashi 2010: Fig. 30) only with a dark anterior stripe, with sparse and rather short setulae; postgonite (Hayashi 2010: Fig. 32) apically swollen spathulata Hayashi 
8. Cercus (Hayashi 2010: Fig. 16) with a group of long setulae; posterior part of surstylus (Hayashi 2010: Fig. 16) curved perpendicularly, with long and sinuate hairs; epiphallus thin and distinctly sinuate dolichodasys Hayashi

- Cercus only with short setulae; surstylus simple; epiphallus thick and straight or indistinctly sinuate

9. Male sternite 5 with posteromedial projection

- Male sternite 5 without posteromedial projection

10. Male sternite 5 (Fig. 1g) with a pair of thin, setulose posteromedial projections, a pale narrow area in the middle and a row of 6 spine-like short setae posterior to the pale area; postgonite (Fig. 1c) sinuate, apically tapering differentialis $\mathrm{Su}$, sp. $\mathbf{n}$.

- Male sternite 5 (Hayashi 2010: Fig. 23) with a pair of thick, setose posteromedial projections, dark along posteromedial incision, with sparse and short setulae; postgonite (Hayashi 2010: Fig. 25) almost straight, apically bootshaped monticola Hayashi

11. Male sternite 5 (Hayashi 2009: Fig. 4) with a pale, large, oval area in the middle; ventral margin of surstylus (Hayashi 2009: Fig. 5) with small setulae; apical half of postgonite (Hayashi 2009: Fig. 6) not swollen; epiphallus (Hayashi 2009: Fig. 6) long and thick, apically tapering australis Hayashi

- Male sternite 5 (Roháček 1983: Fig. 1041) with a pale, small, semicircular area in the middle; ventral margin of surstylus (Roháček 1983: Fig. 1038) with short setae and 2 thick posterior setae; apical half of postgonite (Roháček 1983: Fig. 1037) swollen; epiphallus (Roháček 1983: Fig. 1037) short and thin, apically pointed calcarifera (Roháček)

Acknowledgements. We thank Prof. Mao-Ling Sheng, the Forest Pest Control Station of the State Forestry Administration, China, for his generous loan of specimens, and also
Mr. Yi-Fan Liu, University of Illinois at Urbana-Champaign, U. S. A., for providing valuable references. This work was supported by the National Nature Science Foundation of China (No. 30770252, No. 31071965) and the special funds of Shanxi Zhongtiaoshan Mountains Scientific Research Project.

\section{References}

Collin, J. E. 1902: Four new species of Diptera (Fam. Borboridae) found in Britain. — The Entomologist's Monthly Magazine 38: 55-61.

Hayashi, T. 1989: The genus Opalimosina (s.str.) Rohácek, 1983 from Pakistan (Diptera, Sphaeroceridae). Japanese Journal of Sanitary Zoology. Supplement 40: 61-64.

Hayashi, T. 2009: A new species of the genus Opalimosina Roháček (Diptera, Sphaeroceridae) from the Oriental and Australasian Regions. - The Japan Society of Medical Entomology and Zoology 60(3): 221-224.

Hayashi, T. 2010: The genus Opalimosina Roháček (Diptera, Sphaeroceridae) from Japan. - The Japan Society of Medical Entomology and Zoology 61(3): 309 319.

Papp, L. 1991: Oriental Limosininae: new species and records (Diptera, Sphaeroceridae). - Acta Zoologica Hungarica 37: 225-251.

Marshall, S. A., Roháček, J., Dong H. \& Buck, M. 2011: The state of Sphaeroceridae (Diptera: Acalyptratae): a world catalog update covering the years 2000-2010, with new generic synonymy, new combinations, and new distributions. - Acta Entomologica Musei Nationalis Pragae 51(1): 217-298.

Roháček, J. 1983: A monograph and re-classification of the previous genus Limosina Macquart (Diptera, Sphaeroceridae) of Europe. Part II. - Beiträge zur Entomologie, Berlin 33: 3-195.

Roháček, J., Marshall, S. A., Norrbom, A. L., Buck, M., Quiros, D. I. \& Smith, I. 2001: World catalog of Sphaeroceridae (Diptera). — Slezské zemské Museum, Opava, 414 pp.

Smith, I. P. \& Marshall, S. A. 2004: A review of the New World genus Pterogramma Spuler and a revision of the Pterogramma sublugubrinum group (Diptera: Sphaeroceridae: Limosininae). - Contributions in Science, Natural History Museum of Los Angeles County 499: 1-163.

Su L.-X. 2011: Lesser Dung Flies. — Liaoning University Press, Shenyang. 229 pp. 